\title{
EL IMPACTO DEL MODELO «UN ORDENADOR POR NIÑO» EN LA EDUCACIÓN PRIMARIA: UN ESTUDIO DE CASO
}

\author{
(THE IMPACT OF THE «ONE LAPTOP PER CHILD» MODEL IN PRIMARY \\ EDUCATION: A CASE STUDY)
}

Daniel Losada Iglesias

José Miguel Correa Gorospe

Lorea Fernández Olaskoaga

Universidad del País Vasco (UPV/EHU)

DOI: $10.5944 / e d u c X X 1.17515$

Cómo referenciar este artículo/How to reference this article:

Losada Iglesias, D.; Correa Gorospe, J. M. y Fernández Olaskoaga, L. (2017). El impacto del modelo «un ordenador por niño» en la Educación Primaria: Un estudio de caso. Educación XX1, 20(1), 339-361, doi: 10.5944/educXX1.17515

Losada Iglesias, D.; Correa Gorospe, J. M. \& Fernández Olaskoaga, L. (2017). El impacto del modelo «un ordenador por niño» en la Educación Primaria: Un estudio de caso. [The impact of the "one laptop per child" model in primary education: A case study]. Educación XX1, 20(1), 339-361, doi: 10.5944/educXX1.17515

\section{RESUMEN}

El presente artículo analiza los efectos de los modelos 1:1 para la integración de las TIC en las aulas de los centros escolares de Educación Primaria. Estos modelos se caracterizan por la dotación de un ordenador a cada estudiante (One Laptop Per Child) para el proceso de aprendizaje. Con este objetivo, se ha construido un cuestionario para identificar las expectativas y visiones del profesorado implicado en la iniciativa 1:1 en la Comunidad Autónoma del País Vasco (España) en la que participaron 364 docentes. Las dimensiones recogidas giraron en torno al impacto en la práctica docente tanto para el profesorado como para el alumnado. Los resultados más relevantes obtenidos informan que la mayor dotación de recursos e infraestructura para el aula ha supuesto una oportunidad para el reciclaje y el perfeccionamiento docente, así como una mayor motivación del alumnado en su aprendizaje. No obstante, se concluye que existe una falta de un impacto sustancial, tanto organizativo y pedagógico, en los procesos de enseñanzaaprendizaje. 


\section{PALABRAS CLAVE}

Tecnología Educativa; Política Escolar; Prácticas Educativas; Educación Primaria.

\section{ABSTRACT}

This paper analyzes the effects of 1:1 models for the integration of ICT in the classrooms of primary education schools. These models are characterized by the provision of One Laptop Per Child (OLPC) for the learning process. To this end, we constructed a questionnaire to identify the expectations and visions of teachers involved in the 1:1 initiative in the Basque Autonomous Community (Spain) attended by 364 teachers. The dimensions listed focused on the impact of teaching practices and the daily life of the school both for teachers and for students. The most relevant results report that the largest allocation of resources and infrastructure for the classroom has been an opportunity for recycling and teacher training, as well as increased student motivation in learning. However, the report concludes that there is a lack of substantial impact, both in terms of organizational and pedagogical processes in teaching and learning.

\section{KEYWORDS}

Educational Technology; School Policy; Educational Practices; Primary Education.

\section{INTRODUCCIÓN}

Desde la aparición de los primeros ordenadores en el aula, una de las inquietudes de pedagogos y políticos ha sido valorar la integración de las Tecnologías de la Información y la Comunicación (En adelante TIC) en el sistema educativo. Esta temática se ha convertido en un problema de investigación altamente tratado en la literatura científica (Area, 2005; Condie y Munro, 2007; Culp, Hawkins y Honey, 1999). Entre los investigadores de este fenómeno educativo han ido poco a poco evolucionando las preguntas de investigación. Aunque se siguen planteando cuestiones sobre la influencia que tienen las herramientas y los recursos tecnológicos en el aula, el enfoque actual que utilizan está más centrado en la competencia digital necesaria para la sociedad del conocimiento. De este modo se reivindica el protagonismo metodológico de la tecnología en la escuela del siglo XXI. (Dede, 2011; Voogt y Roblin, 2012). Durante estos últimos años se han publicado muchos estudios sobre la interpretación que hace el profesorado sobre las TIC, sobre 
el mejor modo para integrarlas en los procesos de aprendizaje, así como sobre qué efectos tiene el cambio tecnológico en otros aspectos del proceso educativo, tales como el desarrollo curricular, la organización escolar y los procesos de comunicación (Area, 2005; Cuban, 2001; Culp et al., 1999).

Detrás de estos análisis subyace el interrogante de conocer en qué medida influyen estas nuevas herramientas en la vida diaria de las escuelas. La mayoría de los estudios (Condie y Munro, 2007) concluyen que estas herramientas tecnológicas pueden tener una incidencia significativa en los procesos de enseñanza-aprendizaje, en la organización escolar y en la comunicación con los agentes educativos.

Es evidente que la introducción de las TIC ha estimulado la búsqueda de nuevos caminos para el aprendizaje dentro del contexto educativo y ha permitido un nuevo impulso a la pedagogía. En este sentido, en la revisión de la literatura realizada al respecto por Condie y Munro (2007), la mayoría de los alumnos y de los maestros valoran la introducción de estas nuevas herramientas en las aulas escolares como un avance positivo, gracias a que mejoran la motivación, el rendimiento y en definitiva, el proceso de enseñanza-aprendizaje.

La intensidad y la consistencia de esta mejora educativa a través de las TIC está mediatizada por una serie de factores facilitadores y obstáculos que modulan su eficiencia (Balanskat, Blamire, y Kefala, 2006; BECTA, 2004; Claro, Nussbaum, López, \& Díaz, 2013; Drent y Meelissen, 2008; Ely, 1999; Foon y Brush, 2007; Losada, Karrera, y Jiménez de Aberasturi, 2012; Mumtaz, 2000; Rudd et al., 2009; Tong y Trinidad, 2005; Zhao, Pugh, y Sheldon, 2002). Un repaso a los estudios sobre el impacto de las TIC en las Escuelas en Europa (Balanskat et al., 2006) permite delimitar tres ejes clave donde se desarrollan estas potencialidades y limitaciones.

En un primer nivel (Nivel Micro) se sitúa el docente. Su competencia digital (BECTA, 2004; Vanderlinde y van Braak, 2011; Zhao et al., 2002), así como sus creencias (Ertmer, 2005) y sus actitudes (Sipilä, 2010) son factores clave para un impacto positivo de las TIC en el contexto escolar.

En un nivel intermedio (Nivel Meso) se encuentra la escuela. Los centros educativos condicionan enormemente la implementación y el uso de las TIC por parte del profesorado a través del liderazgo ejercido y de su modelo de organización y planificación de recursos personales y materiales (BECTA, 2004; Claro, Nussbaum, López, \& Díaz, 2013; Drent y Meelissen, 2008; Ely, 1999; Mumtaz, 2000; Rudd et al., 2009; Tong y Trinidad, 2005; Zhao et al., 2002). 
La legislación en materia de educación (Nivel Macro) es el último factor clave a tener en cuenta. Las políticas educativas ejercen una gran influencia sobre la labor del docente y sobre la institución escolar. Los planes y programas diseñados por las administraciones educativas facilitan $\mathrm{u}$ obstaculizan la implementación y el uso de las herramientas digitales en las escuelas (Balanskat et al., 2006; Voogt, Knezek, Cox, Knezek, y Brummelhuis, 2011). Dentro de este marco legal se establece el funcionamiento del Sistema Educativo de cada país o territorio y la organización de los respectivos planes de estudios. Por lo tanto, estas normativas recogen la visión particular sobre la educación y sobre las TIC en los centros escolares. Asimismo, establecen un modelo de dotación de recursos y de formación del profesorado propio.

En su base existe siempre una filosofía y un planteamiento teórico que sustentan la interpretación que realiza la administración educativa ante la sociedad de la información y la comunicación actual. A su vez, estas políticas educativas TIC parecen influir de forma decisiva en la práctica del centro, quizás no en el sentido que quisiera el legislador, pero en definitiva tienen su impacto (Sancho, Petry, Domingo, Müller, y Giró, 2012).

Desde los años 80 del siglo pasado, las diferentes administraciones (europeas, nacionales y autonómicas) han desarrollado estas iniciativas y programas para promover el uso y la integración masiva de las TIC en educación. Estas directrices buscan ser un factor decisivo para un eficaz impacto tecnológico en el contexto escolar, que disminuya las barreras tanto en relación al profesorado como en relación al centro.

A nivel nacional como autonómico, estas políticas TIC han tenido como resultado más relevante la creación de por lo menos un aula informática con acceso a Internet en todos los centros educativos y en algunos casos la dotación de varios recursos tecnológicos (Proyectores...) y computadoras en las aulas ordinarias. Este hecho ha supuesto un impacto limitado en la mejora significativa y generalizada en los procesos de enseñanza-aprendizaje.

Actualmente, con la puesta en escena de modelos 1:1 se ha abierto una nueva línea de actuación tanto a nivel estatal (Escuela 2.0) como en la Comunidad Autónoma Vasca (En adelante CAV) a través de una nueva organización espacial de las TIC en base al modelo de inmersión tecnológica del alumnado (Fernández, Correa, y Losada, 2011; Correa, Losada, y Fernández, 2012). Este modelo 1:1 se basa en la dotación de una computadora por alumno y se asienta en los principios basados en el acceso universal a las tecnologías tanto dentro como fuera del ámbito escolar, en el fomento del aprendizaje colaborativo y en el desarrollo de cierto grado de autonomía. 
Este modelo de inmersión tecnológica está extendiéndose con rapidez principalmente por Europa, Asia y América (Warschauer, 2006). Entre los muchos ejemplos se pueden destacar el Plan Ceibal de Uruguay, el Plan Magallanes de Portugal o el Programa «Conectad.Igualdad» de Argentina. Estas iniciativas han traído consigo una gran inversión de fondos públicos y privados, sin evidencia clara acerca de su relación coste-efectividad. Sin embargo, se han realizado estudios preliminares sobre el efecto en los centros escolares de esta presencia masiva de tecnología. En algunos estudios se concluye que ha habido un impacto positivo en el desarrollo de las competencias TIC del alumnado y en el logro de una mayor equidad en la superación de la brecha digital (Valiente, 2010). No obstante, otros estudios similares realizados sobre la eficacia del programa Escuela 2.0 apuntan que esta iniciativa no ha tenido los efectos didácticos deseados en el modo de organizar y gestionar los procesos de enseñanza-aprendizaje (Santiago, Navaridas y Repáraz, 2014). En definitiva, no se observan mejoras evidentes en el aprendizaje de las áreas curriculares, ni transformación significativa en las estrategias metodológicas del profesorado.

En la CAV, esta propuesta del modelo 1:1 se denomina «Eskola 2.0» y se estructura en tres ejes fundamentales. El primer eje se basa en la dotación de recursos y lleva implícito el modelo 1:1 puesto que transforma la organización espacial de las aulas mediante su digitalización. Este planteamiento pretende ser un avance cualitativo en relación a las políticas pretéritas educativas TIC basadas en la dotación de aulas informáticas centralizadas o en su defecto, en la implementación de ordenadores y periféricos en aulas ordinarias. La digitalización del aula supone garantizar el acceso ubicuo a un "netbook» por parte de cada estudiante. Asimismo, a las aulas inmersas en esta iniciativa se les dota de acceso a Internet mediante una red inalámbrica y se les instala una Pizarra Digital Interactiva.

El segundo eje se basa en la modificación de las prácticas docentes y en la creación de diferentes materiales educativos acordes con la Internet actual. El objetivo que se busca es la mejora de la calidad de los procesos de enseñanza-aprendizaje mediante los postulados de la Web 2.0 o Web social (O’Reilly, 2005). Una web que promueve un usuario activo y protagonista, que crea, discute y comparte a través de innumerables servicios y herramientas.

Esta convergencia de medios digitales mejora el potencial de la comunicación dentro del aula y provee al alumnado de nuevas fórmulas a la hora de transformar la información en conocimiento. Asimismo, los servicios y herramientas web abren la puerta al aprendizaje colaborativo gracias a las nuevas posibilidades de participación y de interacción en diversos espacios compartidos. 
Para llevar a cabo esta «educación 2.0» es necesario integrar una «metodología 2.0» que rompa los roles tradicionales del profesor y del alumno. El docente debe convertirse en el guía y en el dinamizador del proceso de aprendizaje de su alumnado. Teniendo en cuenta este nuevo papel, el profesorado debe llevar a cabo iniciativas que fomenten la participación activa de sus estudiantes.

Para hacer frente al reto de integrar curricularmente la web social en las escuelas vascas, la iniciativa «Eskola 2.0" promueve un tercer eje basado en la formación del profesorado. Esta formación está basada en el modelo teórico TPACK (Mishra y Koehler, 2006) que reivindica que para que exista un uso adecuado de la tecnología en la escuela, el docente debe combinar la compresión y la negociación entre conocimientos tecnológicos, pedagógicos y disciplinares en un contexto determinado. Esta interrelación compleja y contextualizada debe regirse en un conocimiento y un manejo por parte del docente de aspectos relacionados con el tema a tratar, de diferentes estrategias y técnicas didácticas, así como de diversas herramientas tecnológicas actuales (Loveless, 2011).

Es evidente que existen algunos efectos positivos de los Modelos 1:1. No obstante, debido a que los estudios rigurosos son más bien escasos, se necesitan más pruebas sobre cómo se usan las TIC dentro de las clases y sobre cuál es el grado de consecución de los objetivos de estas iniciativas (Penuel, 2006). Debido a esto, el objetivo perseguido en esta investigación educativa es analizar los efectos del modelo 1:1 en las aulas de los centros escolares de Educación Primaria de la CAV en base a las expectativas y visiones de su profesorado.

\section{MÉTODO}

\section{Participantes}

El universo objeto de estudio de esta investigación descriptivo-exploratoria ha sido el profesorado 5. y 6. de Educación Primaria de la CAV que imparte docencia en las aulas digitalizadas de la iniciativa «Eskola 2.0. Se ha realizado un muestreo no probabilístico de corte incidental debido a la dificultad de acceso a los individuos de la población. No obstante, se ha procurado garantizar la representatividad mediante la amplia difusión realizada de la investigación entre la población objeto de estudio.

Según datos del EUSTAT (Instituto Vasco de Estadística), en el curso escolar 2010/2011 llevaban a cabo labores docentes en la Educación Pri- 
maria un total de 7.121 profesores de la red pública. Por lo tanto, se puede estimar que un número no superior a 2.500 docentes, entre maestros tutores y maestros especialistas, intervienen en alguna de las 1.043 aulas digitalizadas de 5. y 6. de primaria en centros públicos (Consejo Escolar de Euskadi, 2013).

Tabla 1.

Estadísticos Descriptivos de los Profesores Participantes en el Cuestionario en Términos de Frecuencias y Porcentajes

\begin{tabular}{c|c|c|c}
\hline \multirow{4}{*}{ Sexo } & & Frecuencia & $\%$ \\
\cline { 2 - 3 } & Varón & 109 & 29.9 \\
& Mujer & 249 & 68.4 \\
Edad & No válido & 6 & 1.6 \\
& 55 o más años & 57 & 15.4 \\
& $45-54$ años & 210 & 57.7 \\
Experiencia & 35-44 años & 60 & 16.5 \\
docente & $25-34$ años & 37 & 9.6 \\
& 21 o más años & 230 & 63.2 \\
& $11-20$ años & 88 & 24.2 \\
& $0-10$ años & 46 & 12.6 \\
\hline
\end{tabular}

La previsión del tamaño de la muestra fue de 334 maestros en base a un nivel de confianza del $95 \%$ y un error muestral previsto de $\pm 5 \%$. Gracias a la colaboración voluntaria y desinteresada de profesores de aulas digitalizadas de Educación Primaria se ha conformado una muestra amplia y representativa de la población a estudiar superior a la prevista. Se ha logrado la participación en el cuestionario (Tabla 1) de un total de 364 profesores de $5 .^{\circ}$ y $6 .^{\circ}$ de Educación Primaria para la obtención de sus visiones y expectativas ante la iniciativa autonómica basada en el Modelo 1:1.

\section{Instrumentos}

El instrumento de recogida de datos utilizado en este estudio empírico ha sido el cuestionario. Su diseño lo han llevado a cabo 65 investigadores que participan en una investigación sobre los modelos 1:1 financiada por el Ministerio de Ciencia e Innovación del Gobierno de España. Esta herramienta ad hoc está construida en varias etapas a partir de la definición operacional del constructo que subyace del modelo 1:1 de integración de las TIC y de las características propias de la implantación en las diferentes comunidades autónomas de España. Este cuestionario tiene como finali- 
dad obtener información sobre los usos, las expectativas, las opiniones y las valoraciones del profesorado en relación a las iniciativas de inmersión tecnológica implantadas en España.

Con el objeto de fortalecer inicialmente la validez de contenido del instrumento, se sometieron a criterio de varios jueces expertos en la realidad de la integración TIC y con las sugerencias recibidas se redujeron y/o ajustaron los ítems. La estructura conceptual del cuestionario está dividida en 6 dimensiones, contenidas en 32 preguntas con respuestas de elección simple o múltiple, abiertas, así como escalas valorativas tipo Likert.

La validez interna del instrumento ha sido demostrada. El nivel de consistencia interna de todo el cuestionario obtenido mediante el cálculo del coeficiente Alfa de Cronbach ha sido excelente $(\alpha=.946)$. Asimismo, los índices de fiabilidad obtenidos en cada una de sus dimensiones del cuestionario han sido superiores a $\alpha=.73$.

La dimensión «Datos identificativos» contiene las variables independientes de este estudio. Se estructura en 9 ítems sobre las características personales y profesionales del profesor. Por un lado, se recoge la edad y el sexo del encuestado. Por otro lado, se formulan preguntas sobre diversos aspectos profesionales: Tipo de centro donde trabaja, experiencia docente previa, curso y ciclo en el que desarrolla su actividad, así como áreas curriculares en las que imparte.

En la segunda dimensión denominada «TIC y práctica docente en el aula», a través de 6 ítems se indaga sobre los recursos TIC que dispone como profesor en su aula, su actitud ante los mismos, así como la frecuencia de uso y el tipo de actividades que realiza. El tipo de agrupamiento que utiliza en las tareas realizadas con TIC es también una información requerida. Por último, el efecto de la tecnología en la práctica docente está recogida en una pregunta. La consistencia interna de la dimensión ha sido de $\alpha=.776$.

La tercera dimensión denominada «TIC y centro escolar» consta de 3 ítems y tiene como objeto conocer su valoración acerca del impacto de la iniciativa «Eskola 2.0» en la escuela donde trabaja. Asimismo, existen cuestiones relacionadas con el papel del coordinador TIC del centro. La fiabilidad de la dimensión fue de $\alpha=.737$.

La cuarta dimensión denominada «TIC y alumnado» se compone de 4 preguntas que tiene como objetivo recopilar información sobre los recursos tecnológicos que dispone en el hogar el alumnado y el uso que hace de los mismos. Asimismo, en este apartado se recoge la opinión sobre las habilida- 
des digitales de sus estudiantes y el impacto de las mismas sobre sus estrategias de aprendizaje. La consistencia interna de este factor ha sido de $\alpha=.933$.

La quinta dimensión denominada «TIC y profesorado» recopila información mediante 4 ítems sobre la frecuencia de uso de herramientas tecnológicas por parte del profesorado en el ámbito doméstico. Asimismo, recoge cuestiones acerca de las demandas formativas que a juicio del encuestado son necesarias para la utilización de las TIC. La fiabilidad de la dimensión ha sido de $\alpha=.888$.

La sexta y última dimensión denominada «Valoración de la iniciativa 1:1» explora la opinión del docente encuestado en relación a esta política. Mediante 6 preguntas se pretende obtener el grado de información sobre la iniciativa que dispone y el tipo de impacto que prevé a medio plazo. Por último, los aspectos positivos y negativos de la iniciativa son recogidos a través de preguntas abiertas. La consistencia interna del factor ha sido de $\alpha=.906$.

\section{Procedimiento}

En este estudio empírico la recogida de datos se ha realizado mediante un cuestionario online, gracias al apoyo técnico ofrecido por el Instituto de Tecnología Educativa dependiente del Ministerio de Educación del Gobierno de España. Durante el primer semestre de 2011 el profesorado de la red vasca de enseñanza ha tenido a su disposición dicho instrumento para su participación voluntaria.

\section{Análisis de datos}

La aplicación informática utilizada ha sido el paquete estadístico IBM SPSS en su versión 19. Tras la codificación de los ítems, el etiquetado de las variables y la introducción de los datos en este software, en todas las respuestas recogidas se han realizado diferentes análisis estadísticos. Tanto en las respuestas de elección simple o múltiple, así como en las escalas valorativas tipo Likert se han realizado estadísticos descriptivos y de frecuencia, así como inferenciales (Chi cuadrado y ANOVA).

\section{RESULTADOS}

Tras los análisis estadísticos pertinentes solo se han encontrado diferencias significativas en el ítem que recoge información sobre el efecto de la 
tecnología en el aprendizaje en relación a la edad del docente. No obstante, a partir de la diversidad y la significatividad existente en la muestra se puede describir la realidad de la implantación y el efecto del modelo 1:1 en la CAV. Para tal fin, los resultados obtenidos se han clasificado en función de 4 cuestiones a responder acerca de este modelo de integración de las TIC en el aula.

\section{¿Cómo es la práctica docente en el aula con las TIC?}

Se observa una frecuencia de utilización de los diferentes recursos didácticos en la que destaca el uso diario de los libros de texto $(61,3 \%)$, de la pizarra tradicional $(53,6 \%)$ y del cuaderno del alumno, de las enciclopedias y de otros documentos en papel (50,3\%). Asimismo, menos de la mitad de los profesores manejan todos los días los ordenadores $(43,1 \%)$ e Internet $(44,2 \%)$ para el desarrollo de su práctica educativa. Sin embargo, la frecuencia de uso mayoritaria de la pizarra digital interactiva (el 36\% utiliza varios días a la semana) y de los medios audiovisuales tradicionales como el video (el 29,7\% utiliza alguna vez en el curso) no es diaria.

Las actividades realizadas mayoritariamente con las herramientas tecnológicas existentes dentro del aula por los encuestados (Figura 1) son: la petición de búsquedas de información en Internet al alumnado (93,7\%), la realización de trabajos con procesador de texto $(78,6 \%)$ y la resolución de actividades y ejercicios online $(68,1 \%)$. Asimismo, otra de las acciones más realizadas es la explicación de los contenidos de los temas $(76,1 \%)$ apoyada en la Pizarra Digital Interactiva. Otro tipo de actividades con las TIC son escasamente realizadas.

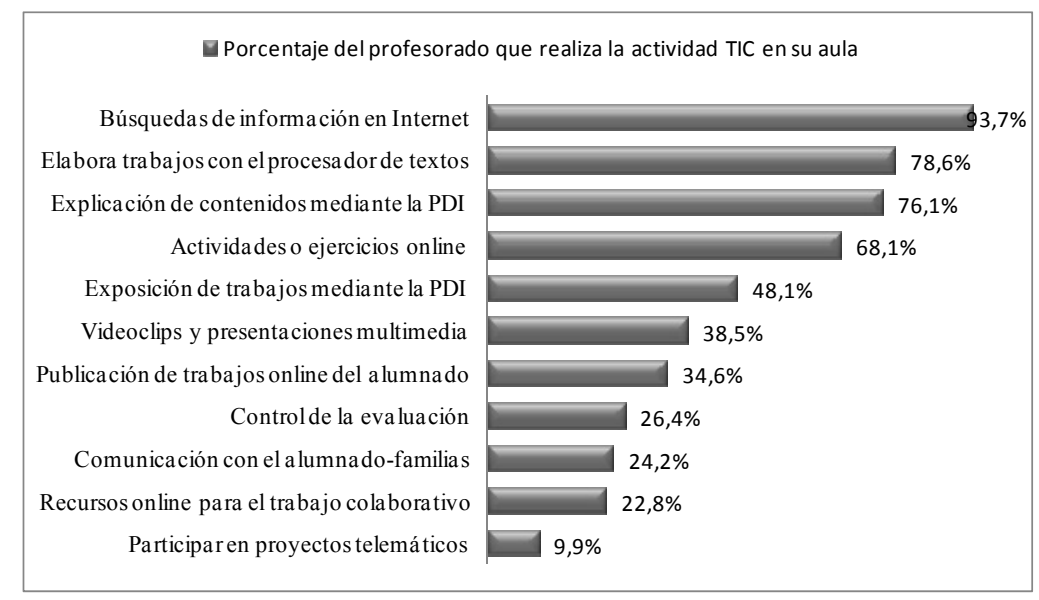

Figura 1. Tipos de acciones desarrolladas con las TIC 
En relación al modo de agrupar y organizar el alumnado para realizar las tareas anteriormente mencionadas, más de la mitad del profesorado $(59,1 \%)$ sitúa todos los días o varios días a la semana a sus estudiantes individualmente con el fin de trabajar con el ordenador. La organización basada en pequeños grupos que trabajan autónomamente es mucho menos utilizada, con un 53,9\% de docentes que la llevan a cabo esporádicamente. Por último, la utilización de la tecnología en gran grupo es muy poco habitual. Más de la mitad del profesorado $(58,3 \%)$ casi nunca o nunca agrupa al alumnado de esta forma.

Sobre el impacto que están teniendo las TIC en su práctica docente, los encuestados han respondido mayoritariamente $(83,8 \%)$ que existe una mayor motivación e implicación del alumnado en las actividades del aula. Asimismo, el profesorado participante en este cuestionario $(80,8 \%)$ considera que estas herramientas están provocando un cambio o una transformación en su metodología de enseñanza. El desarrollo de nuevos métodos de enseñanza (metodología por proyectos, aprendizaje cooperativo, investigación en el aula, proyectos intercentros,...) y la reorganización de los tiempos, los espacios y los agrupamientos dentro del aula son efectos reconocidos por más de la mitad de los docentes. Eso sí, la pérdida del protagonismo del libro de texto, incluyendo su menor relevancia en el proceso educativo, solo es reconocida por el $44,5 \%$ de los encuestados.

Únicamente un porcentaje reducido de los profesores considera negativamente el impacto de las herramientas tecnológicas en su práctica en el aula. La sensación de inseguridad y confusión del maestro en su utilización $(30,8 \%)$, la pérdida de tiempo y la distracción del alumnado $(13,7 \%)$ y la sensación de que el esfuerzo no compensa $(12,1 \%)$ son respuestas poco frecuentes entre los encuestados.

Otro de los temas tradicionales asociados a la implantación de las TIC en los procesos de enseñanza-aprendizaje son los materiales digitales y su disponibilidad. Los encuestados en su mayoría están totalmente o bastante de acuerdo en que la administración debe crear y publicar mucha más cantidad de materiales didácticos online $(75,7 \%)$ y debe potenciar sitios web como Agrega, Educared, Aulablog y similares, puesto que consideran que son buenos recursos para encontrar materiales o contenidos digitales $(71,2 \%)$.

Por último, con los resultados obtenidos se pone en cuestión dentro del trabajo en el aula la utilización de las herramientas y servicios web 2.0. Solamente el $26,9 \%$ del profesorado considera conveniente una utilización masiva de blogs, wikis, redes sociales, servidores de video... en su práctica docente. 


\section{¿Cómo se organizan las TIC en el centro escolar y cómo es la comunicación con el entorno?}

Tras la implantación de la iniciativa «Eskola 2.0», el impacto más evidente que ha sufrido la escuela radica en la mejora sustancial de recursos e infraestructura TIC disponibles, tanto en cantidad como en calidad (57.7\%). Asimismo, el papel del coordinador TIC dentro de la escuela es considerado por la mayoría del profesorado encuestado $(88,8 \%)$ como muy necesario o bastante necesario. Desgraciadamente, un $15,4 \%$ de los mismos no disponen en su centro de esta figura.

Entre las funciones mejor valoradas destacan la orientación al profesorado en el desarrollo de la docencia con TIC $(63,2 \%)$, la administración y la actualización de los recursos y herramientas informáticas $(65,7 \%)$, así como la organización de la formación del profesorado sobre TIC en el centro $(57,1 \%)$. Asimismo, alrededor de la mitad de los profesores informan que los coordinadores TIC ofrecen información sobre los contenidos o materiales digitales en la web y administran los recursos extranet del centro (web, blog, sitio web, portal noticias, wiki, red social,...) en sus centros.

Sin embargo, no se ha registrado un enriquecimiento de las vías de comunicación con los diferentes miembros de la comunidad escolar y con los agentes educativos externos. Los profesores encuestados reconocen que la comunicación online entre el profesorado del centro $(76,9 \%)$, con las familias $(79,9 \%)$ y con el entorno social, cultural y económico $(75,8 \%)$, así como el contacto con otros centros educativos a través de Internet $(85,4 \%)$ y con los servicios educativos de la administración $(73,1 \%)$ no ha sufrido una mejora sustancial con la implantación de esta iniciativa.

\section{¿Cómo usa las TIC el alumnado?}

En relación al hogar, casi tres cuartas partes de estos docentes $(73,1 \%)$ tienen la mayoría o todo el alumnado con ordenador e Internet. Entre la división en tres tareas básicas que se pueden realizar con un ordenador en sus casas, los docentes opinan mayoritariamente que la frecuencia de uso es diaria o casi todos los días de la semana para el ocio, el juego y la diversión $(74,7 \%)$, así como para comunicarse con sus amigos $(65,4 \%)$. Sin embargo, El uso es más esporádico a la hora de realizar las tareas escolares. Los estudiantes $(72 \%)$ utilizan ocasionalmente las TIC para el estudio.

A través de la valoración de los profesores encuestados se ha obtenido la competencia digital de los estudiantes. Los valores recogidos se han situado entre 1 (Nada) a 5 (Mucho). Las medias obtenidas proporcionan un perfil 
competencial (Figura 2) basado en el manejo técnico de los diferentes aparatos y hardware y en la habilidad para buscar y localizar información en Internet.

-Media del profesorado sobre la percepción de la habilidad digital de su alumnado.

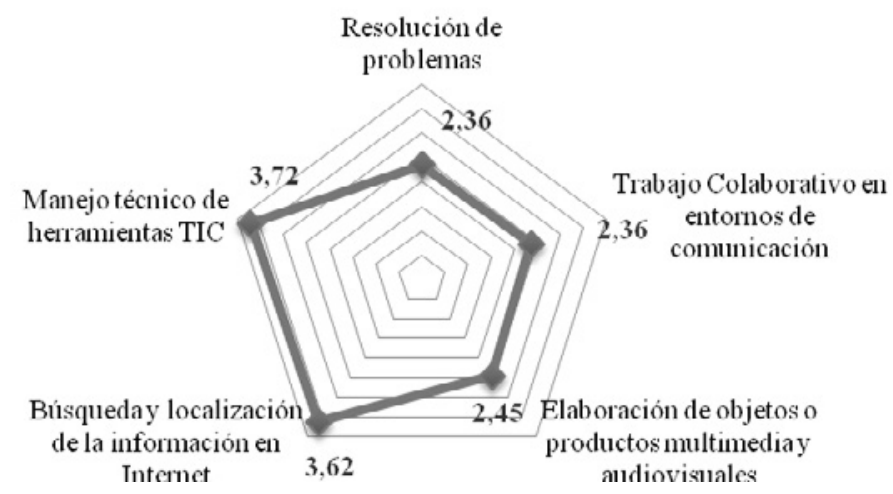

Figura 2. Perfil competencial del alumnado ante las TIC

Sin embargo, los datos obtenidos muestran un distanciamiento significativo por debajo del valor medio (2,5 puntos) de la escala Likert en habilidades TIC importantes. En general, el profesorado considera que sus estudiantes están poco capacitados para trabajar colaborativamente en entornos de comunicación (blogs, wikis...), para resolver problemas y tomar decisiones, así como para elaborar objetos o productos multimedia mediante las TIC.

En relación al contexto escolar, los efectos más significativos (Tabla 2) que tienen las TIC en el aprendizaje son el incremento de la motivación y la implicación en las tareas de clase, el desarrollo de la competencia digital y la mejora de la capacidad de gestión de la información, así como la facilitación a la hora de buscar en distintas fuentes y recursos. En estos dos últimos ítems existen diferencias significativas en los docentes de edades comprendidas entre 35 y 54 años.

Tabla 2.

Diferencias de Medias en el Efecto de las TIC en el Aprendizaje en Relación a la Edad del Docente

\begin{tabular}{l|l|r|r|r|r|r}
\hline & & $\mathbf{2 5 - 3 4}$ & $\mathbf{3 5 - 4 4}$ & $\mathbf{4 5 - 5 4}$ & \multicolumn{1}{c}{$\mathbf{5 5}$} & Total \\
\cline { 2 - 7 } Están más motivados e implicados con la & $\mathrm{N}$ & 37 & 70 & 210 & 57 & 364 \\
tarea de clase & Media & 3.35 & 3.98 & 3.83 & 3.54 & 3.76 \\
& DT & 1.549 & .965 & 1.015 & 1.415 & 1.152 \\
& $\mathrm{~F}$ & & & & & 3.318 \\
& Sig. & & & & & $.020^{*}$ \\
\hline
\end{tabular}




\begin{tabular}{|c|c|c|c|c|c|c|}
\hline & & $25-34$ & $35-44$ & 45-54 & '55 & Total \\
\hline $\begin{array}{l}\text { Han desarrollado la competencia digital } \\
\text { y gestión de la información }\end{array}$ & \begin{tabular}{|l} 
N \\
Media \\
DT \\
F \\
Sig. \\
\end{tabular} & $\begin{array}{r}37 \\
3.14 \\
1.456\end{array}$ & $\begin{array}{r}70 \\
3.66 \\
.993\end{array}$ & $\begin{array}{r}210 \\
3.34 \\
1.052\end{array}$ & $\begin{array}{r}57 \\
3.16 \\
1.360\end{array}$ & $\begin{array}{r}364 \\
3.34 \\
1.147 \\
2.054 \\
.052 \\
\end{array}$ \\
\hline $\begin{array}{l}\text { Buscan información y utilizan distintas } \\
\text { fuentes }\end{array}$ & \begin{tabular}{|l|}
$\mathrm{N}$ \\
Media \\
$\mathrm{DT}$ \\
$\mathrm{F}$ \\
Sig. \\
\end{tabular} & $\begin{array}{r}37 \\
2.92 \\
1.299\end{array}$ & $\begin{array}{r}70 \\
3.57 \\
.851\end{array}$ & $\begin{array}{r}210 \\
3.38 \\
1.034\end{array}$ & $\begin{array}{r}57 \\
2.96 \\
1.322\end{array}$ & $\begin{array}{r}364 \\
3.30 \\
1.104 \\
4.918 \\
.002 *\end{array}$ \\
\hline $\begin{array}{l}\text { Han adquirido mejor los conocimientos / } \\
\text { contenidos de la materia }\end{array}$ & \begin{tabular}{|l}
$\mathrm{N}$ \\
Media \\
DT \\
F \\
Sig. \\
\end{tabular} & $\begin{array}{r}37 \\
2.54 \\
1.238\end{array}$ & $\begin{array}{r}70 \\
3.02 \\
.983\end{array}$ & $\begin{array}{r}210 \\
3.00 \\
.923\end{array}$ & $\begin{array}{r}57 \\
2.72 \\
1.320\end{array}$ & $\begin{array}{r}364 \\
2.91 \\
1.046 \\
2.941 \\
.033 *\end{array}$ \\
\hline Ha mejorado su rendimiento & \begin{tabular}{|l}
$\mathrm{N}$ \\
Media \\
$\mathrm{DT}$ \\
$\mathrm{F}$ \\
Sig. \\
\end{tabular} & $\begin{array}{r}37 \\
2.46 \\
1.304\end{array}$ & $\begin{array}{r}70 \\
3.07 \\
.918\end{array}$ & $\begin{array}{r}210 \\
2.92 \\
1.034\end{array}$ & $\begin{array}{r}57 \\
2.79 \\
1.333\end{array}$ & $\begin{array}{r}364 \\
2.88 \\
1.105 \\
2.604 \\
.052 \\
\end{array}$ \\
\hline $\begin{array}{l}\text { Trabajan más en equipo y de forma colabo- } \\
\text { rativa }\end{array}$ & \begin{tabular}{|l|} 
N \\
Media \\
DT \\
F \\
Sig.
\end{tabular} & $\begin{array}{r}37 \\
2.43 \\
1.405\end{array}$ & $\begin{array}{r}70 \\
2.98 \\
.983\end{array}$ & $\begin{array}{r}210 \\
2.76 \\
1.137\end{array}$ & $\begin{array}{r}57 \\
2.65 \\
1.261\end{array}$ & $\begin{array}{r}364 \\
2.75 \\
1.167 \\
1.873 \\
.134\end{array}$ \\
\hline $\begin{array}{l}\text { Saben utilizar y expresarse a través de dis- } \\
\text { tintos lenguajes (Visual, sonoro...) }\end{array}$ & \begin{tabular}{|l} 
N \\
Media \\
DT \\
F \\
Sig. \\
\end{tabular} & $\begin{array}{r}37 \\
2.38 \\
1.320\end{array}$ & $\begin{array}{r}70 \\
2.80 \\
1.086\end{array}$ & $\begin{array}{r}210 \\
2.83 \\
1.115\end{array}$ & $\begin{array}{r}57 \\
2.35 \\
1.445\end{array}$ & $\begin{array}{r}364 \\
2.70 \\
1.201 \\
3.498 \\
.016 *\end{array}$ \\
\hline Saben expresarse y comunicarse mejor & \begin{tabular}{|l|}
$\mathrm{N}$ \\
Media \\
$\mathrm{DT}$ \\
$\mathrm{F}$ \\
Sig.
\end{tabular} & $\begin{array}{r}37 \\
1.86 \\
1.110\end{array}$ & $\begin{array}{r}70 \\
2.27 \\
.989\end{array}$ & $\begin{array}{r}210 \\
2.31 \\
.962\end{array}$ & $\begin{array}{r}57 \\
2.19 \\
1.093\end{array}$ & $\begin{array}{r}364 \\
2.24 \\
1.008 \\
2.165 \\
.092\end{array}$ \\
\hline
\end{tabular}

\section{¿Cuál es la valoración y sus expectativas con respecto al modelo 1:1?}

Solo algo menos de un cuarto de los encuestados $(22,8 \%)$ considera acertada la actual política educativa sobre TIC. Sin embargo, una mayoría significativa de la muestra $(62,1 \%)$ apoya la política de inversiones basada en la digitalización de su aula. Los profesores valoran positivamente dispo- 
ner de una Pizarra Digital Interactiva, de conexión a Internet y de un ordenador por alumno dentro del aula. Sin embargo, no existe consenso sobre la pertinencia de extender la iniciativa a los restantes ciclos de la Educación Primaria.

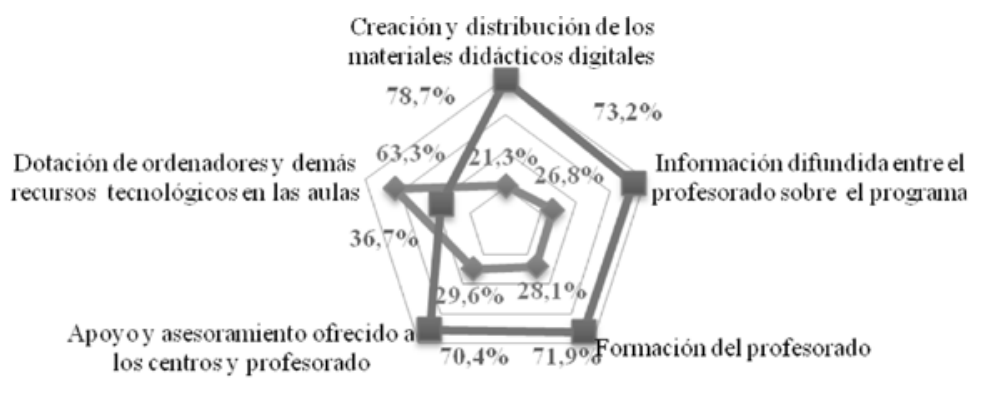

$\rightarrow$ Muy bien o bien $\quad=$ Regular, mal o muy mal

Figura 3. Valoración de la política de implantación del modelo 1:1 en CAV

Con respecto al impacto a medio plazo de la iniciativa «Eskola 2.0», la opinión del profesorado encuestado es casi unánime $(96,2 \%)$ puesto que intuyen que tendrá algún efecto destacable. En este sentido, aproximadamente 8 de cada 10 docentes consideran que aumentará notablemente la cantidad de tecnología disponible en los centros y aulas, proporcionará mayor formación al profesorado en el uso de las TIC y fomentará la innovación en la metodología docente.

Por último, a modo resumen (Figura 3) se puede afirmar que la iniciativa «Eskola 2.0» solo aprueba en la dotación de recursos tecnológicos en el aula $(63,3 \%)$. Lamentablemente, esta política suspende ampliamente en aspectos tan variados como la información difundida entre el profesorado $(73,2 \%)$, en la formación desarrollada $(71,9 \%)$, en el apoyo y asesoramiento ofrecido a los centros $(70,4 \%)$ y en la creación y distribución de los objetos digitales educativos $(78,7 \%)$.

\section{DISCUSIÓN}

El problema de investigación planteado en este estudio ha obligado a recabar información sobre el impacto organizativo y pedagógico del modelo OLPC. El cuestionario aplicado al profesorado ha reportado una primera radiografía del modelo de acceso ubicuo de ordenadores para cada alumno en la CAV y su impacto en la mejora de la calidad en la educación. 
Se puede concluir que la irrupción del modelo 1:1 no ha supuesto la presencia de la filosofía 2.0 ni del Modelo TPACK en las aulas. En este sentido, tanto el modo de utilización de las herramientas y de los materiales digitales, así como su frecuencia de uso describen un planteamiento didáctico tradicional. La preponderancia del libro de texto y del cuaderno del alumno no ha variado. La frecuencia de utilización de los ordenadores e Internet dentro del aula es inferior con respecto a este tipo de tecnologías clásicas.

Es verdad que se observan ciertos guiños a la renovación de herramientas dentro del aula. El profesorado incluye a veces en su planteamiento didáctico la utilización de ordenadores para la búsqueda y el tratamiento de la información, así como para la resolución de actividades y ejercicios individuales online. Pero, estos leves cambios en la metodología docente, fundamentados principalmente en el uso de navegadores web y procesadores de texto, no implican la tan ansiada transformación de la práctica profesional. $\mathrm{El}$ aprendizaje y el trabajo colaborativo del alumnado no se garantizan.

La iniciativa actual no ha fomentado todavía el uso de herramientas tecnológicas actuales para la compartición de espacios de trabajo y para la comunicación entre personas. Asimismo, los docentes continúan promoviendo el agrupamiento individual a la hora de usar las TIC. Hay que recordar que entre sus bases teóricas existe una apuesta firme y clara hacia la Web social y hacia una metodología 2.0. Es evidente que ante este planteamiento tan claro y respaldado por una inversión económica tan importante, esta política educativa debiera ya haber empezado a dar sus frutos.

Pero tras la recogida de datos, se debe concluir que actualmente dentro de las aulas no existe una utilización rica y variada de las TIC que saque partido a este potencial de comunicación y transformación de la información en conocimiento. El rol clásico del profesorado basado en la explicación de los contenidos y de las lecciones, aunque sea con soporte de la Pizarra Digital Interactiva, no es suficiente para lograr el protagonismo activo y participativo del alumnado, ni la interacción entre pares.

Aunque existen muy pocos cambios en la metodología utilizada para la práctica docente, se perfilan unas cuantas mejoras. El profesorado ha logrado mitigar una serie de barreras tradicionales a nivel de profesor. Se observa que se ha generalizado la percepción de los beneficios de las herramientas digitales en el contexto escolar, sobre todo a la hora de motivar e implicar al alumnado. Asimismo, la mejora de la confianza en sí mismo reportada puede provocar nuevos planteamientos y ahondar más allá de pequeñas modificaciones, asumiendo una verdadera transformación de su práctica docente. 
En esta misma línea, la implantación del modelo 1:1 no ha supuesto una transformación organizativa y de comunicación hacia planteamientos colaborativos en los centros escolares. Esta política sigue perpetuando una visión tecnocentrista de la integración de las TIC. El aumento de las herramientas tecnológicas en la escuela no ha supuesto una mejora significativa en los procesos comunicativos y de interacción en la institución con los diversos agentes educativos con los que se relaciona. Aunque ha habido un mayor número de ordenadores y periféricos en los centros, incluso una mejor conexión a Internet, este hecho no ha supuesto una mejora sustancial de la manera de estar en contacto con el alumnado, las familias y demás agentes de la comunidad escolar.

Asimismo, en la línea con otros estudios (McGarr y McDonagh, 2013), esta investigación ha puesto de manifiesto que la figura del coordinador TIC dentro del centro escolar sigue cumpliendo funciones eminentemente técnicas frente a otras de mayor calado o con mayor influencia pedagógica. Una verdadera ruptura con la gramática escolar exige disponer de recursos personales formados y dispuestos a liderar en el reto de la integración tecnológica. Como evidencian Tondeur, Cooper, y Newhouse (2010), los coordinadores TIC deben incluirse formalmente dentro de las estructuras de decisión de la escuela para un buen impacto en la integración de las TIC. Pero, difícilmente se logrará un impacto positivo de lo tecnológico, si su tarea principal sigue siendo la resolución de problemas técnicos y no una verdadera reflexión sobre dónde está su centro escolar y hacia dónde se dirige. Seguramente, esta falta de planificación dificulta enormemente la orientación al profesorado en el desarrollo de la docencia con estos nuevos recursos y la consecución de la tan ansiada transformación de la práctica docente dentro de las aulas.

Por último, es necesario concluir que las evidencias recogidas informan que el modelo OLCP no tiene actualmente el efecto deseado en base a lo esperado por sus impulsores dentro de la administración educativa vasca. Falta una reflexividad sobre la justificación pedagógica del uso de las TIC en el aula por parte del profesorado (Fernández, Losada, Correa, 2014). Sin embargo, las expectativas a medio plazo del profesorado auguran una posibilidad de cambio metodológico en su práctica docente. Se constata que los docentes consideran una buena oportunidad para poder tener al alcance un mayor número de recursos tecnológicos y una mejor infraestructura en sus centros escolares, así como para poder reciclarse y mejorar ante lo digital en su trabajo. Pero, ¿será suficiente para mejorar la calidad en la educación?

Lo más probable es que la innovación pedagógica con TIC, aunque sea de modo aislado, será promovida por este modelo 1:1. No obstante, no se puede olvidar que políticas educativas TIC anteriores, en base a su novedad, 
impulsaron a cierto número de docentes y de escuelas a hacer algo diferente y a adaptarse a las nuevas demandas sociales (De Pablos, Area, Valverde y Correa, 2010).

A partir de las experiencias y evaluaciones de los proyectos de 1:1, tanto en países desarrollados como en aquellos en desarrollo, se evidencia que estas herramientas tecnológicas al servicio del alumnado serán un fracaso en la transformación de las prácticas educativas del centro, si no vienen acompañadas de un proyecto educativo (Area, 2011). Si se pretende conseguir un uso significativo y eficaz de las TIC, las escuelas deben garantizar el ajuste entre sus concepciones pedagógicas con la forma que utilizan estas tecnologías en las aulas (de Koster, Kuiper, y Volman, 2012). Por lo tanto, una visión compartida sobre la innovación es esencial para el logro de una implementación efectiva de las tecnologías (Kirkland \& Sutch, 2009; Law et al., 2008; Fullan, 2001). Solo así se podrá tener un impacto transformador sobre la gramática escolar reguladora de los modelos implícitos de la práctica docente.

\section{AGRADECIMIENTOS:}

*Proyecto «Las políticas de un «ordenador por niño» en España. Visiones y prácticas del profesorado ante el programa Escuela 2.0. Un análisis comparado entre comunidades autónomas». EDU2010-17037. MICINN 2010-2014.

*Grupo de investigación consolidado por el Sistema Universitario Vasco «ELKARRIKERTUZ: Innovación educativa con tecnologías digitales: cambio tecnológico y transformaciones culturales y educativas» (IT563-13). Convocatoria 2013-2018). 


\section{REFERENCIAS BIBLIOGRÁFICAS}

Area, M. (2005). Tecnologías de la Información y Comunicación en el Sistema Escolar: Una revisión de las líneas de investigación. Revista Electrónica de Investigación y Evaluación Educativa, 11(1), 3-25.

Area, M. (2011). Los efectos del modelo $1: 1$ en el cambio educativo en las escuelas. Evidencias y desafíos para las políticas iberoamericanas. Revista Iberoamericana de Educación, 56, 49-74.

Balanskat, A., Blamire, R. \& Kefala, S. (2006). The ICT Impact Report. A. review of studies of ICT impact on schools in Europe European Schoolnet. European Communities. Recuperado de http://goo.gl/gUxEbQ

BECTA. (2004). A review of the research literature on barriers to the uptake of ICT by teachers. Retrieved from http:// goo.gl/0nMbE

Claro, M., Nussbaum, M., López, X., \& Díaz, A. (2013). Introducing 1 to 1 in the Classroom: A Large-scale Experience in Chile. Educational Technology and Society, 16(3), 315-328.

Condie, R. \& Munro, B. (2007). The impact of ICT in schools - a landscape review. Retrieved from http://goo.gl/ UjBYVA

Consejo Escolar de Euskadi (2013). La Educación en Euskadi: Informe 20102012. Vitoria-Gasteiz: Servicio Central de Publicaciones del Gobierno Vasco.

Correa, J.M., Losada, D. y Fernández, L. (2012). Políticas educativas y prácticas escolares de integración de las tecnologías en las Escuelas del País Vasco: Voces y cuestiones emergentes. Campus Virtuales: Revista Científica Iberoamericana de Tecnología Educativa, 1(1), 21-30.
Cuban, L. (2001). Oversold and Underused: Computers in the Classroom. Harvard, Massachusetts: Harward University Press.

Culp, K.M., Hawkins, J. \& Honey, M. (1999). Review paper on educational technology research and development. Recuperado de http://goo.gl/CAx49t

de Koster, S., Kuiper, E. y Volman, M. (2012). Concept-guided development of ICT use in 'traditional' and 'innovative' primary schools: what types of ICT use do schools develop? Journal of Computer Assisted Learning, 28(5), 454-464. doi: 10.1111/j.13652729.2011.00452.x

De Pablos, J., Area, M., Valverde, J. y Correa, J. M. (Eds.). (2010). Políticas Educativas y Buenas Prácticas con TIC. Barcelona: Grao.

Dede, C. (2011). Reconceptualizing technology integration to meet the challenges of educational transformation. Journal of Curriculum and Instruction, 5(1), 4-16. doi: 10.3776/joci.2011. v5n1p4-16

Drent, M. \& Meelissen, M. (2008). Which factors obstruct or stimulate teacher educators to use ICT innovatively? Computers and Education, 51(1), 187199.

Ely, D. P. (1999). Conditions That Facilitate the Implementation of Educational Technology Innovations. Educational Technology, 39(6), 23-27.

Ertmer, P. A. (2005). Teacher pedagogical beliefs: The final frontier in our quest for technology integration? Educational Technology Research and Development, 53(4), 25-39. doi: 10.1007/ BF02504683 
Fernández, L., Correa, J. M. \& Losada, D. (2011). OLPC project in the Basque Country: Eskola 2.0. Procedia - Social and Behavioral Sciences, 15, 22072213. doi:10.1016/j.sbspro.2011.04.081

Fernández, L., Losada, D., y Correa, J. M. (2014). Análisis intercasos de prácticas TIC en las aulas de Educación Primaria con un modelo 1:1. Profesorado: Revista de Curriculum Y Formación Del Profesorado, 18(3), 27-40.

Foon, K. y Brush, T. (2007). Integrating technology into K-12 teaching and learning: current knowledge gaps and recommendations for future research. Educational Technology Research and Development, 55(3), 223-252. doi: 10.1007/s11423-006-9022-5

Fullan, M. (2001). The new meaning of educational change. New York, NY: Teachers College Press.

Kirkland, K., \& Sutch, D. (2009). Overcoming the barriers to educational innovation: A literature review. Retrieved from https://goo.gl/AgiUCW

Law, N., Pelgrum, W. J. \& Plomp, T. (Eds.). (2008). Pedagogy and ICT Use in Schools around the World. Hong Kong: Springer.

Losada, D., Karrera, I. \& Jiménez de Aberasturi, E. (2012). Factors Facilitating Successful Educational Innovation with ICT in Schools. Revista de Psicodidáctica, 17(1), 113-134.

Loveless, A. (2011). Technology, pedagogy and education: reflections on the accomplishment of what teachers know, do and believe in a digital age. Technology, Pedagogy and Education, 20(3), 301-316. doi: 10.1080/1475939X.2011.610931

McGarr, O., \& McDonagh, A. (2013). Examining the role of the ICT coordinator in Irish post-primary schools. Techno- logy, Pedagogy and Education, 1-16. doi: 10.1080/1475939X.2012.755132

Mishra, P. \& Koehler, M.J. (2006). Technological pedagogical content knowledge: A framework for integrating technology in teachers' knowledge. Teachers College Record, 108(6), 1017-1054.

Mumtaz, S. (2000). Factors Affecting Teachers' Use of Information and Communications Technology: a review of the literature. Journal of Information Technology for Teacher Education, 9(3), 319-342.

O'Reilly, T. (2005). What is Web 2.0? Design patterns and business models for the next generation of software. Retrieved from http://goo.gl/r4yY

Penuel, W.R. (2006). Implementation and Effects Of One-to-One Computing Initiatives: A Research Synthesis. Journal of Research on Technology in Education, 38, 329-348.

Rudd, P., Teeman, D., Marshall, H., Mundy, E., White, K., Lin, Y. \& Cardozo, V. (2009). Harnessing Technology Schools Survey 2009: analysis report. Recuperado de http://goo.gl/Q7YIwf

Santiago, R., Navaridas, F., \& Reparaz, C. (2014). La Escuela 2.0: La percepción del docente en torno a su efiacia en los centros educativos de La Rioja. Educación XX1, 17(1), 243-270.

Sancho, J. M., Petry, P. P., Domingo, L., Müller, J. y Giró, X. (2012). El Instituto La Mallora. Una apuesta por la integración de las TIC. In J.M. Sancho y C. Alonso (Eds.), La fugacidad de las politicas, la inercia de las prácticas (pp. 61-85). Barcelona: Octaedro.

Sipilä, K. (2010). The impact of laptop provision on teacher attitudes towards ICT. Technology, Peda- 
gogy and Education, 19(1), 3-16. doi: 10.1080/14759390903579257

Tondeur, J., Cooper, M. y Newhouse, C. P. (2010). From ICT coordination to ICT integration: a longitudinal case study. Journal of Computer Assisted Learning, 26(4), 296-306. doi: 10.1111/j.13652729.2010.00351.x

Tong, K.P. \& Trinidad, S.G. (2005). Conditions and Constraints of Sustainable Innovative Pedagogical Practices Using Technology. International Electronic Journal For Leadership in Learning, 9(3). Recuperado de http://goo.gl/ Fpytta

Valiente, O. (2010). 1-1 in Education: Current Practice, International Comparative Research Evidence and Policy Implications. OECD Education Working Papers, 44. doi: http://dx.doi. org/10.1787/5kmjzwfl9vr2-en

Vanderlinde, R. \& van Braak, J. (2011). A new ICT curriculum for primary education in flanders: Defining and predicting teachers' perceptions of in- novation attributes. Educational Technology and Society, 14(2), 124-135.

Voogt, J., Knezek, G., Cox, M., Knezek, D. \& Brummelhuis, A. (2011). Under which conditions does ICT have a positive effect on teaching and learning? A Call to Action. Journal of computer assisted learning. doi: 10.1111/j.13652729.2011.00453.x

Voogt, J. y Roblin, N.P. (2012). A comparative analysis of internatinal frameworks for 21st centuruy competences: implications for national curriculum policies. Journal of $\mathrm{Cu}$ rriculum Studies, 44(3), 299-321. doi: $10.1080 / 00220272.2012 .668938$

Warschauer, M. (2006). Laptops and literacy: Learning in the wireless classroom. New York: Teacher"s College Press.

Zhao, Y., Pugh, K. \& Sheldon, S. (2002). Conditions for classroom technology innovations. Teachers College Record, 104(3), 482-515. 


\section{PERFIL ACADÉMICO Y PROFESIONAL DE LOS AUTORES}

Daniel Losada Iglesias. Doctor en Ciencias de la Educación y profesor agregado del departamento de Didáctica y Organización Escolar de la Universidad del País Vasco. Es miembro del grupo de investigación consolidado por el Sistema Universitario Vasco «ELKARRIKERTUZ: Innovación educativa con tecnologías digitales: cambio tecnológico y transformaciones culturales y educativas» (IT563-13). Sus principales líneas de investigación son la tecnología educativa y la formación de profesorado. Cuenta con publicaciones en diferentes revistas nacionales e internacionales.

José Miguel Correa Gorospe. Catedrático de Escuela Universitaria del Departamento de Didáctica y Organización Escolar de la Universidad del País Vasco. Es el investigador principal del grupo de investigación consolidado por el Sistema Universitario Vasco «ELKARRIKERTUZ: Innovación educativa con tecnologías digitales: cambio tecnológico y transformaciones culturales y educativas» (IT563-13). Ha dirigido proyectos de investigación competitivos nacionales y autonómicos. Su actividad investigadora se centra en la construcción de la identidad docente y en el estudio de la integración de las TIC en la educación. Cuenta con publicaciones en diferentes revistas nacionales e internacionales.

Lorea Fernández Olaskoaga. Profesora del departamento de Didáctica y Organización Escolar de la Universidad del País Vasco y doctora en Ciencias de la Educación. Es miembro del grupo de investigación consolidado por el Sistema Universitario Vasco «ELKARRIKERTUZ: Innovación educativa con tecnologías digitales: cambio tecnológico y transformaciones culturales y educativas» (IT563-13). Su línea de investigación se centra en el campo de las Tecnologías de la Información y la Comunicación. Cuenta con diferentes publicaciones en obras colectivas nacionales.

Dirección de los autores: Daniel Losada Iglesias

Departamento de Didáctica y Organización Escolar

Facultad de Educación, Filosofía y Antropología

Plaza Oñati, 3

20018 Donostia-San Sebastián

E-mail: daniel.losada@ehu.eus

José Miguel Correa Gorospe

Departamento de Didáctica y Organización Escolar 
Facultad de Educación, Filosofía y Antropología

Plaza Oñati, 3

20018 Donostia-San Sebastián

E-mail: jm.correagorospe@ehu.eus

Lorea Fernández

Departamento de Didáctica y Organización

Escolar

Facultad de Educación, Filosofía y Antropología

Plaza Oñati, 3

20018 Donostia-San Sebastián

E-mail: lorea.fernandez@ehu.eus

Fecha Recepción del Artículo: 18. Febrero. 2014

Fecha modificación Artículo: 06. Mayo. 2014

Fecha Aceptación del Artículo: 15. Mayo. 2014

Fecha Revisión para Publicación: 13. Junio. 2016 
